\title{
Transatlantica
}

Revue d'études américaines. American Studies Journal

\section{Annie Lechenet. Jefferson-Madison, Un débat sur la République.}

Paris : PUF, 2003, collections « Philosophies ». 128 pages.

\section{Marie-Jeanne Rossignol}

\section{(QpenEdition}

\section{Journals}

Édition électronique

URL : http://journals.openedition.org/transatlantica/852

DOI : $10.4000 /$ transatlantica.852

ISSN : 1765-2766

Éditeur

AFEA

Référence électronique

Marie-Jeanne Rossignol, « Annie Lechenet. Jefferson-Madison, Un débat sur la République. »,

Transatlantica [En ligne], 1 | 2005, mis en ligne le 22 avril 2006, consulté le 29 avril 2021. URL : http://

journals.openedition.org/transatlantica/852 ; DOI : https://doi.org/10.4000/transatlantica.852

Ce document a été généré automatiquement le 29 avril 2021.

\section{c) (i) $९$}

Transatlantica - Revue d'études américaines est mis à disposition selon les termes de la licence Creative Commons Attribution - Pas d'Utilisation Commerciale - Pas de Modification 4.0 International. 


\section{Annie Lechenet. Jefferson-Madison, Un débat sur la République.}

Paris : PUF, 2003, collections « Philosophies ». 128 pages.

Marie-Jeanne Rossignol

1 Annie Léchenet est une philosophe, auteure d'une thèse sur la citoyenneté chez Thomas Jefferson. Pour cet ouvrage au format imposé (128 pages), les PUF lui ont confié la mission plus ambitieuse encore consistant à présenter les idées de Jefferson et de Madison. L'angle choisi est plutôt original : alors qu'il est de tradition d'associer les deux hommes, fondateurs du parti démocrate-républicain dans les années 1790, puis proches collaborateurs politiques tout le reste de leur existence, Annie Léchenet montre comment leurs idées sur la République s'opposent en fait au tout début de la République fédérale, pour aboutir ensuite à une vision commune, mais renouvelée, de la République.

2 La lecture de l'ouvrage est parfois décevante: le format très condensé réduit la bibliographie à une vingtaine d'«éléments bibliographiques » et oblige l'auteure à résumer une réflexion riche et complexe ; la méthode choisie peut s'avérer déroutante également pour le lecteur-historien qui voit mêlées sur la même page des références aux écrits de Jefferson de 1786 comme de 1816. Est-ce le même Jefferson? Le contexte est quasiment absent, et toute la discussion sur la liberté et la participation politique fait l'économie de la question des femmes ou de l'esclavage: position peut-être compréhensible pour une philosophe, mais la science politique et l'histoire peuvent difficilement faire abstraction de ces dimensions. Le libéralisme politique de Jefferson correspond, après la « Révolution de 1800 » qui marque son élection selon certains, à un rétrécissement de l'espace public pour les femmes et les Africains-Américains. [Voir The Revolution of 1800: Democracy, Race, and the New Republic. James Horn, Jan Allen Lewis et Peter S. Onuf, eds. Charlottesville : University Press of Virginia, 2002.]

3 Pourtant ce petit livre aux analyses très personnelles a un énorme mérite : à un moment où l'historiographie américaine a en grande partie délaissé le débat sur le républicanisme, Annie Léchenet reprend cette problématique avec les outils du philosophe et lui redonne une grande actualité en reprenant, sur un plan plus modeste, 
le fil du récit là où l'avait laissé Gordon S. Wood, dans son livre célèbre et traduit en français La création de la République américaine 1776-1787 (1969. Paris : Belin, 1991). [Voir le chapitre IV de Canada / Etats-Unis depuis 1770. C. Fohlen, J. Heffer et F. Weil eds. Paris : PUF, 1997 ; Daniel T. Rodgers, "Republicanism : the Career of a Concept ", Journal of American History. Vol. 79, ${ }^{\circ} 1$, June 1992.]

Comme chacun le sait maintenant, entre autres depuis la large diffusion des thèses de John G. A Pocock (Le moment machiavélien 1975. Paris : PUF, 1997), le modèle républicain qui domine la pensée des révolutionnaires américains, c'est la République dite "classique " héritée de la pensée anglaise: un dispositif politique, nous rappelle A. Léchenet, fondé d'abord sur l'équilibre des pouvoirs, chaque pouvoir représentant un des ordres de la nation. Dépourvus de Roi et d'aristocratie, les anciens « insurgés » vont refonder cette idée classique de la République en l'appuyant uniquement sur le peuple: c'est là une «invention de la République américaine » qu'ont notée et commentée Wood ou Denis Lacorne dans son ouvrage du même titre de 1991.

5 Toute la difficulté des années 1776-1787 - ceci est bien connu et Annie Léchenet ne s'y appesantit pas - sera de trouver moyen d'équilibrer cette nouvelle République, puisque cet équilibre ne peut s'appuyer sur les différents ordres de la société. Confronté à l'exigence populaire de liberté héritée de la Révolution, comme aux craintes des possédants face aux dérives démocratiques, James Madison élabore une Constitution qui équilibre liberté (pouvoir populaire majoritaire) et justice (droits des minorités, donc des possédants). L'édifice républicain permet de fragmenter et de dissoudre les différences d'«intérêts » qui sont naturellement appelées à s'exprimer dans ce type de régime.

6 En revanche, Thomas Jefferson, selon Annie Léchenet, est bien plus radicalement attaché à la notion de participation populaire que soucieux d'équilibre des factions et des pouvoirs : pour lui, le citoyen ne saurait mal utiliser sa liberté. Un gouvernement est «plus ou moins républicain selon la proportion dans laquelle il intègre dans sa composition plus ou moins de cet ingrédient qu'est l'action directe des citoyens » (cit. de Jefferson p. 58). Jefferson, écrit A. Léchenet, a sur ce point un raisonnement proche de Rousseau (p.60), piste qu'elle n'élabore malheureusement pas. La République de Jefferson ne saurait être une construction passive, qui vise seulement à empêcher un groupe de faire prédominer ses intérêts; au contraire, Jefferson fait confiance aux intérêts exprimés par le vote, et les représentants doivent respecter les vœux de leurs électeurs, non s'élever au-dessus d'eux. Implicite dans de telles analyses, le lien entre Jefferson et les Anti-fédéralistes, critiques de la Constitution, est lui aussi peu exploité et expliqué dans le livre, ce que l'on peut regretter.

7 Eloignée des citoyens, la construction constitutionnelle ne risque-t-elle pas de revêtir «le masque de l'oligarchie» (p.74) s'interroge Annie Léchenet? C'est effectivement ainsi que ces mêmes Anti-fédéralistes perçurent la Constitution en 1787, puis d'autres auteurs jusqu'à aujourd'hui (p.89). Sans véritablement entrer dans ce débat bien connu, Annie Léchenet explique que Madison concevait son architecture constitutionnelle surtout comme un moyen de parvenir au compromis : ainsi réduisait-il le politique au juridique et faisait-il reposer son édifice sur le sens moral, la vertu des représentants fédéraux que le système constitutionnel aurait débarrassés de leurs préjugés locaux ou partisans. La pensée du Père de la Constitution s'inscrit alors dans la tradition républicaine anglaise. Mais à la différence de nombreux penseurs, Madison voit rapidement ses idées s'incarner dans un fonctionnement concret et se pose alors la 
question: la vertu motivera-t-elle effectivement les décisions impartiales, quoique politiques, de ce nouveau gouvernement?

Dès les débuts de la République fédérale, dès 1789, Madison est amené, rappelle A. Léchenet, à douter de l'efficacité du système qu'il a contribué à édifier (p.91): le pouvoir n'est pas habité par la recherche du bien public, selon lui, et se met à servir des intérêts oligarchiques. La pensée de Madison va alors se confondre avec celle de Jefferson. "L'invention de la république américaine » a lieu à ce moment-là, encore davantage qu'en 1787 : Jefferson et Madison créent un parti et participent à des luttes partisanes que nul n'imaginait possibles en 1787. Ce parti, explique A. Léchenet, n'a aucun rapport avec les factions et les partis dénoncés par la pensée républicaine anglaise ou le premier Madison : pour ses inventeurs, le parti républicain exprime une vision cohérente et populaire du bien public. Il représente aussi des intérêts - pour Annie Léchenet, il s'agit essentiellement des intérêts agricoles - mais ceux-ci, aux yeux de Jefferson et Madison, se confondent avec le bien public. Le politique ne s'édifie pas au-dessus des intérêts particuliers, mais avec eux: car l'intérêt particulier, bien compris, ne peut rechercher que l'intérêt de tous. Le social, le politique, le juridique et la morale sont des niveaux intimement mêlés et non strictement différenciés dans une République. La République de Jefferson, semble dire Annie Léchenet, est un débat, une recherche permanente de la justice pour tous dans une liberté garantie à chacun, un processus dynamique plus qu'une structure posée une fois pour toutes.

9 L'arrivée de George Bush au pouvoir a quelque peu remis au goût du jour la «Révolution de 1800 », quand Thomas Jefferson et son parti parvinrent au pouvoir et où ils purent prôner davantage de démocratie sans vraiment changer la politique américaine. Sur certains sites web, le " Patriot Act » est comparé aux lois séditieuses votées sous John Adams, prédécesseur de Jefferson et contre lesquelles il s'opposa avec James Madison par les célèbres «Kentucky resolutions ». On découvre au fil du livre d'Annie Léchenet qu'il a également été écrit dans cet esprit: relier les idées des Fondateurs américains aux grands débats sur la démocratie d'aujourd'hui. Mais Jefferson et Madison ne se livrèrent pas seulement à la réflexion politique durant leurs longues vies actives et l'on ne saurait rappeler leur combat pour la liberté sans signaler les nombreux droits qu'ils bafouèrent eux-mêmes.

INDEX

Thèmes : Recensions

\section{AUTEUR}

MARIE-JEANNE ROSSIGNOL

Université Paris 7-Denis Diderot 